\title{
A Model Problem with the Coexistence of Stochastic and Integrable Behaviour
}

\author{
Maciej Wojtkowski \\ Institute of Mathematics, Warsaw University, PL-00-901 Warszawa, PKiN IX p., Poland
}

\begin{abstract}
A one parameter family of piecewise linear measure preserving transformations of a torus which can be viewed as a perturbation of the twist mapping is introduced. Theorems on their ergodic properties for an infinite set of parameters are proved. For some parameters coexistence of stochastic and integrable behaviour is obtained.
\end{abstract}

\section{Introduction}

The celebrated theorem of Kolmogorov (KAM theory) on the conservation of quasiperiodic motions in classical dynamical systems gave rise to the problem of ergodic properties in the regions where invariant tori are destroyed. Since that time no examples were provided in which the situation could be clarified. Katok [1] gave a construction of a smooth Bernoulli diffeomorphism on the twodimensional disc which is equal to the identity on the boundary. Hence this transformation can be sewed together with whatever one likes. It proves that in principle quasiperiodic motions and the Bernoulli component can coexist in a smooth dynamical system.

In this paper we give an example of a one parameter family of transformations of a two-dimensional torus for which the coexistence of stochastic and integrable behaviour can be proved for an infinite set of parameters. All transformations are piecewise linear (with two pieces) and hence are not smooth. This family can be viewed as a perturbation of an integrable system - the twist mapping (considered on the torus and not on an annulus as usual). So our example is to some extent given a priori.

The technique used for proving stochastic behaviour is much the same as in [2]. By stochastic behaviour we mean almost hyperbolicity: the existence almost everywhere (in some domain) of local expanding and contracting fibres which form absolutely continuous foliations. By classical methods this allows us to prove that the domain consists of sets of positive measure on which some power of the transformation is a $K$-system. Actually in our case the methods of Ornstein can be 
applied to prove that it is Bernoulli [3]. Ergodicity cannot be obtained from general considerations and we are able to prove it only in some cases.

It turns out that the analysis of ergodic properties should be started from "big" perturbations. In [2] it was proved that for a wide class of "big" piecewise linear perturbations of the twist map the whole system exibits stochastic behaviour. A similar result was obtained much earlier by Oseledec $([4,5])$, but to my knowledge, the details of his results remained unpublished. In this paper we take smaller perturbations and study the appearance of islands of periodic (quasiperiodic) motions. These islands persist for whole intervals of parameters (even up to the twist mapping). We are able to prove the presence of stochastic behaviour only for a sequence of parameters. Moreover it seems quite possible that there is a large set of parameters for which the metric entropy of the system is zero.

Although this work is purely analytical, computer experiments played an important role in the investigation. These calculations were done by Marek Sołtykiewicz whom the author thanks warmly.

\section{Description of the Family of Transformations}

Let $\mathbb{T}^{2}$ be a two-dimensional torus with coordinates $\left(\varphi_{1}, \varphi_{2}\right) \bmod 1$. Let us consider the twist mapping $F_{1}: \mathbb{T}^{2} \rightarrow \mathbb{T}^{2}, F_{1}\left(\varphi_{1}, \varphi_{2}\right)=\left(\varphi_{1}+\varphi_{2}, \varphi_{2}\right)$ and the mapping $F_{2}: \mathbb{T}^{2} \rightarrow \mathbb{T}^{2}$ defined by $F_{2}\left(\varphi_{1}, \varphi_{2}\right)=\left(\varphi_{1}, \varphi_{2}+\varepsilon f\left(\varphi_{1}\right)\right)$ where $\varepsilon \in \mathbb{R}$ and $f$ is the real function $f(t)=\left\{\begin{array}{rrr}-t-\frac{1}{4} & \text { for } & -\frac{1}{2} \leqq t \leqq 0 \\ t-\frac{1}{4} & \text { for } & 0 \leqq t \leqq \frac{1}{2}\end{array}\right.$.

Both $F_{1}$ and $F_{2}$ preserve the Lebesgue measure $d \varphi_{1} d \varphi_{2}$. The object of our study will be the family of transformations $F_{\varepsilon, N}=F_{2} \circ F_{1}^{N}$. We will consider their ergodic properties with respect to the Lebesgue measure. This family can be treated as a perturbation of the twist mapping $F_{1}^{N}$.

We introduce the change of variables

$$
x_{1}=\varphi_{1}, x_{2}=\varphi_{1}+N \varphi_{2} .
$$

In these variables $F_{\varepsilon, N}$ is equal to $T_{A}, T_{A}\left(x_{1}, x_{2}\right)=\left(x_{2},-x_{1}+2 x_{2}+A f\left(x_{2}\right)\right)$ where $A=\varepsilon N$. More precisely the above formulas define a good change of variables on $\mathbb{R}^{2}$ but not on $\mathbb{T}^{2}$. On $\mathbb{T}^{2}$ we have only that $F_{\varepsilon, N}$ covers $N$ to one $T_{A}$ and conjugacy takes place only for $N=1$. However the almost hyperbolic structure of $T_{A}$ lifts to a similar structure for $F_{\varepsilon, N}$, and this allows us to deduce properties of $F_{\varepsilon, N}$ from those of $T_{A}$. We will not do it explicitly and restrict ourselves to the study of $T_{A}$.

Let $S: \mathbb{T}^{2} \rightarrow \mathbb{T}^{2}$ denote the transformation $S\left(x_{1}, x_{2}\right)=\left(x_{2}, x_{1}\right)$. Then one checks that $S \circ T_{A} \circ S=T_{A}^{-1}$. This symmetry relation simplifies the investigation of $T_{A}$. It is called $S$-reversibility (cf. [6]). It is worth mentioning that it holds for arbitrary $f$. $T_{A}$ and $T_{-A}$ are the same up to a change of variables so we assume $A>0$.

We will consider the following fundamental domain in the coordinate plane $\left(x_{1}, x_{2}\right)\left\{\left(x_{1}, x_{2}\right) \mid-\frac{1}{2} \leqq x_{i} \leqq \frac{1}{2}, i=1,2\right\}$. Furthermore let

$$
\mathscr{B}_{ \pm}=\left\{\left(x_{1}, x_{2}\right) \mid 0 \leqq \pm x_{2} \leqq \frac{1}{2}\right\}
$$

and

$$
\mathscr{C}_{ \pm}=\left\{\left(x_{1}, x_{2}\right) \mid 0 \leqq \pm x_{1} \leqq \frac{1}{2}\right\}
$$


We have that $T_{A}$ is linear in $\mathscr{B}_{+}$and $\mathscr{B}_{-}$and $T_{A}\left(\mathscr{B}_{ \pm}\right)=\mathscr{C}_{ \pm}$. In $\mathscr{B}_{ \pm}$there is a fixed point $\left( \pm \frac{1}{4}, \pm \frac{1}{4}\right)$. The differential of $T_{A}$ in $\mathscr{B}_{ \pm}$is given by the matrices $\left[\begin{array}{cc}0 & 1 \\ -1 & 2 \pm A\end{array}\right]$.

Before proceeding with rigorous theorems we will describe properties of $T_{A}$ on a heuristic level. We start with the following important remark. If $T_{A}$ has a periodic orbit $\left\{p, T_{A} p, \ldots, T_{A}^{k} p=p\right\}$ which is elliptic - i.e. the linear part of $T_{A}^{k}$ in $p$ has eigenvalues on the unit circle different from 1 and -1 - then there is a domain $\mathscr{D} \ni p$ such that $T_{A}^{k}$ is a linear rotation in $\mathscr{D}$. The same is true of the domains $T_{A}^{i} \mathscr{D}$, $i=1, \ldots, k-1$. If the angle of rotation is rational $\left(i\right.$.e. of the form $\left.\frac{p}{q} 2 \pi\right)$ then $\mathscr{D}$ is a polygon and $T_{A}^{k}$ is periodic in it; otherwise it is an ellipse. For brevity we will call such a domain an elliptic island.

It is easy to see that when $A$ is an integer all points with rational coordinates are periodic and hence all islands, if there are any, are polygons.

Computer calculations indicate the following picture. For $A \geqq 4$ we have one stochastic "sea" ("filled" with one orbit). For $A<4$ the fixed point $\left(-\frac{1}{4},-\frac{1}{4}\right)$ becomes elliptic and there arises the elliptic island which is an ellipse if $\frac{1}{\pi} \arccos \left(\frac{A}{2}-1\right)$ is irrational, and a polygon otherwise. For $2 \leqq A<4$ outside the island we have apparently a stochastic "sea", in particular there seem to be no more elliptic islands. For $A<2$ the periodic orbit of period $2\left\{\left(\frac{1}{4},-\frac{1}{4}\right),\left(-\frac{1}{4}, \frac{1}{4}\right)\right\}$ becomes elliptic and we have two more elliptic islands. For $1<A<\frac{\sqrt{17}-1}{2}$ there are two elliptic orbits of period 3 [one of them is the orbit of the point $\left(\frac{A+5}{4(A+3)}, \frac{A+1}{4(A+3)}\right)$, the other is symmetric under $\left.S\right]$. For $A$ around 1 we have also two elliptic orbits of period 5. The stochastic "sea" surrounding the increasing number of elliptic islands is divided into two parts apparently by two closed invariant curves for $A$ somewhere between 1.1 and 1.2. For $A<1$ at first the invariant curves are destroyed and then the picture becomes more and more complicated.

The presence of elliptic islands is a matter of straightforward calculations. The problem is to prove mixing properties of the transformation outside the islands. In the following this is done for some values of the parameter $A$.

\section{The Notion of Almost Hyperbolicity}

In this paragraph we specify some abstract theorems which will be used in the following. For simplicity we restrict ourselves to a piecewise linear measure preserving transformations of a torus. To shorten further formulations we introduce the following definition.

Definition. We say that a piecewise linear measure preserving transformation $T: \mathbb{T}^{2} \rightarrow \mathbb{T}^{2}$ is almost hyperbolic in an invariant domain $\mathscr{K} \subset \mathbb{T}^{2}$ iff there are two 
families of disjoint segments passing through almost all points of $\mathscr{K}$, called respectively local contracting and expanding fibres, with the following properties:

Denote by $\gamma^{s}(p)\left(\gamma^{u}(p)\right)$ the local contracting (expanding) fiber passing through $p \in \mathscr{K}$.

(i) $\gamma^{s}(p)$ and $\gamma^{u}(p)$ intersect transversally;

(ii) $T\left(\gamma^{s}(p)\right) \subset \gamma^{s}(T(p))$ and $T^{-1}\left(\gamma^{u}(p)\right) \subset \gamma^{u}\left(T^{-1}(p)\right)$;

(iii) if $p_{1}, p_{2} \in \gamma^{s}(p)\left(\gamma^{u}(p)\right)$ then

$$
\operatorname{dist}\left(T^{n} p_{1}, T^{n} p_{2}\right)<\mathrm{const} \lambda^{n} \quad \text { for } \quad n>0(n<0),
$$

where $\lambda(0<\lambda<1)$ and the constant depend on the fiber.

In [2] it was proved that, in general, a partition into segments is measurable and conditional measures are equivalent to arc length.

By the work of Hopf, Hedlund, Anosov, Sinai, and Ornstein it is now wellestablished that almost hyperbolicity in the above sense leads to strong mixing properties of $T$ in the domain $\mathscr{K}$ (see for instance [7] or for a simple exposition [8]). More exactly there is a family of invariant subsets of positive measure $\mathscr{K}_{i}$, $i=1,2, \ldots, \bigcup_{i=1}^{\infty} \mathscr{K}_{i}=\mathscr{K}$ such that $\left.T\right|_{\mathscr{K}_{\imath}}$ is ergodic. Moreover $\mathscr{K}_{i}=\mathscr{K}_{i}^{1} \cup \ldots \cup \mathscr{K}_{i}^{n_{i}}$, $\mathscr{K}_{i}^{k} \cap \mathscr{K}_{i}^{\ell}=\emptyset$ if $k \neq \ell, T \mathscr{K}_{i}^{\ell}=\mathscr{K}_{i}^{\ell+1}, \ell=1, \ldots, n_{i}-1, T \mathscr{K}_{i}^{n_{i}}=\mathscr{K}_{i}^{1}$ and $\left.T^{n_{i}}\right|_{\mathscr{K}_{i}^{1}}$ is a $K$-system. In particular $\left.T\right|_{\mathscr{K}}$ has countably many ergodic components. In [3] it was checked that in the above situation (piecewise linear almost hyperbolic transformation) Ornstein's method [9] can be applied to prove that actually $\left.T^{n_{i}}\right|_{\mathscr{\kappa}_{1}^{\prime 1}}$ is Bernoulli.

To establish the number of ergodic components one needs more information about $T$. We will use the following criterion of ergodicity.

Theorem 1. Let $T: \mathbb{T}^{2} \rightarrow \mathbb{T}^{2}$ be almost hyperbolic in an invariant domain $\mathscr{K} \subset \mathbb{T}^{2}$ and suppose that for any local expanding and contracting fibres, $\gamma^{u}(p)$ and $\gamma^{s}(q)$ there is $N \geqq 0$ such that $T^{k} \gamma^{u}(p)$ intersects $T^{-\ell} \gamma^{s}(q)$ whenever $k, \ell \geqq N$. Then $T$ and all its powers are ergodic in $\mathscr{K}$.

Proof. This is just the repetition of the classical proof [8]. Let $f: \mathbb{T}^{2} \rightarrow \mathbb{R}$ be a continuous function. We will show that the ergodic mean $f_{+}=\lim _{k \rightarrow \infty} \frac{1}{k} \sum_{i=0}^{k-1} f \circ T^{i}$ is almost constant. It is constant on local contracting fibers (if it exists). Consider the set $\mathscr{X}=\left\{p \in \mathscr{K} \mid f_{+}(p)\right.$ and $f_{-}(p)$ exist and $\left.f_{+}(p)=f_{-}(p)\right\}$ where $f_{-}=\lim _{k \rightarrow \infty} \frac{1}{k} \sum_{i=0}^{k-1} f \circ T^{-i} . \mathscr{X}$ is $T$-invariant and has full measure. So except for a set of contracting fibers of total measure zero, almost all points of a contracting fiber in the sense of arc length belong to $\mathscr{X}$. (Of course we use absolute continuity of the foliation into local contracting fibres.) Suppose $\gamma^{s}(q)$ has the above property. Let $\mathscr{Y}_{\ell}$ be the sum of local expanding fibres which intersect $T^{-\ell} \gamma^{s}(q)$ in the points from $\mathscr{X} . T^{-\ell} \gamma^{s}(q)$ is a broken line. It follows that $\bigcup_{\ell=0}^{\infty} \mathscr{Y}_{\ell}$ has full measure in view of the assumption and because the set of segments intersecting a fixed segment on the set of arc length zero has measure zero (once again absolute continuity). 
$f_{-}$is constant (if it exists) on local expanding fibres. So we obtain that $f_{+}$is constant on $\mathscr{Y}_{\ell}$ for any $\ell \geqq 0$ and hence almost everywhere.

It is easy to see that under the assumptions of Theorem $1 T$ is topologically transitive in $\mathscr{K}$ (if it is continuous).

To establish almost hyperbolicity for $S$-reversible transformations it suffices to find expanding fibres. Then contracting fibres are given by $\gamma^{s}(p)=S\left(\gamma^{u}(S p)\right)$. We will use the following criterion of almost hyperbolicity which we formulate only for $S$-reversible transformations.

Let $\mathscr{K}$ be some domain on a torus not necessarily invariant and suppose that for almost all points $p \in \mathscr{K}$ there is a sector $U(p)$ in the tangent space so that the following properties hold.

(i) $D T_{\mathscr{K}^{\prime}}(U(p)) \subset U\left(T_{\mathscr{K}^{\prime}}(p)\right)$ where $T_{\mathscr{K}}: \mathscr{K} \rightarrow \mathscr{K}$ is the first return map;

(ii) for $v \in U(p)\left\|D T^{n+1} v\right\| \geqq\left\|D T^{n} v\right\|, n \geqq 0$ and for almost all points $p \in \mathscr{K}$ there are $0<\lambda<1$ and $k>0$ such that $\left\|D T^{k} v\right\| \geqq \lambda^{-1}\|v\|$.

Theorem 2. If (i) and (ii) hold then $T$ is almost hyperbolic in $\mathscr{K}_{1}=\bigcup_{i=-\infty}^{+\infty} T^{i}(\mathscr{K})$.

Proof. We give a sketch of the proof. Most of the details can be found in [2].

By the property (i) we can extend the sector bundle $U(p)$ on the whole set $\bigcup_{i=0}^{+\infty} T^{i}(\mathscr{K})$ with preservation of property (i) for $T$ in place of $T_{\mathscr{K}}$ and property (ii). $\bigcup_{i=0}^{+\infty} T^{i}(\mathscr{K})$ is almost equal to $\mathscr{K}_{1}$.

Now we put $U_{n}(p)=D T^{n}\left(U\left(T^{-n} p\right)\right)$. We have obviously $U_{n+1}(p) \subset U_{n}(p)$ $n=0,1, \ldots$ Let $\mathscr{X}_{\lambda, k}=\left\{p \in \mathscr{K}_{1} \mid\left\|D T^{k} v\right\| \geqq \lambda^{-1}\|v\|\right.$ for $\left.v \in U(p)\right\}$ where $0<\lambda<1$ and $k>0$. By property (ii) the sum of the sets $\mathscr{X}_{\lambda, k}$ over all $k>0$ and any sequence $\lambda_{i} \nearrow 1$ is the whole $\mathscr{K}_{1}$. Fix $\mathscr{X}_{\lambda, k}$ with positive measure. We will construct expanding fibres through almost all points from $\mathscr{X}_{\lambda, k}$.

Almost all points from $\mathscr{X}_{\lambda, k}$ return to it with positive frequency under iterates of the map $T^{-k}$. It follows that for almost all points $p \in \mathscr{X}_{\lambda, k}$ there is $0<v<1$ such that

$$
\left\|D T^{k n} v\right\| \geqq \operatorname{const} \lambda^{-v n}\|v\| \quad \text { for } \quad v \in U\left(T^{-k n} p\right), \quad n \geqq 0 .
$$

So there is $0<\lambda_{1}<1$ such that

$$
\left\|D T^{n} v\right\| \geqq \text { const } \lambda_{1}^{-n}\|v\| \quad \text { for } \quad v \in U\left(T^{-n} p\right), \quad n_{0} \geqq 0 .
$$

It follows that for almost all points $U_{\infty}(p)=\bigcap_{n=0}^{\infty} U_{n}(p)$ is a single line. Now we consider a segment through $p$ with the direction of $U_{\infty}(p)$. Under the action of $T^{-1}$ it is contracted exponentially. At the same time by a standard argument almost all points approach singularity lines at a rate not faster than const $n^{-2}$ where $n$ is the number of iterations. So taking a sufficiently short segment we obtain that it never cuts singularity lines. We define $\gamma^{u}(p)$ as the maximal segment with the above property.

Thus $\gamma^{u}(p)$ can be constructed for almost all points $p \in \mathscr{K}_{1}$ and obviously they have the required properties. So $T$ is almost hyperbolic in $\mathscr{K}_{1}$. 


\section{Ergodic Properties of $T_{A}$ for $A \geqq 4$}

In the tangent space to $\mathbb{T}^{2}$ we introduce coordinates $\xi_{i}=d x_{i}, i=1,2$, and the norm $\left\|\left(\xi_{1}, \xi_{2}\right)\right\|=\max \left(\left|\xi_{1}\right|,\left|\xi_{2}\right|\right)$. We define the following bundle of sectors on $\mathbb{T}^{2}$

$$
U(p)=\left\{\begin{array}{lll}
\left\{\left(\xi_{1}, \xi_{2}\right) \mid-a+1+\sqrt{a(a-2)} \leqq \frac{\xi_{1}}{\xi_{2}} \leqq 0\right\} & \text { if } & p \in \mathscr{C}_{-} \\
\left\{\left(\xi_{1}, \xi_{2}\right) \mid 0 \leqq \frac{\xi_{1}}{\xi_{2}} \leqq a+1-\sqrt{a(a+2)}\right\} & \text { if } & p \in \mathscr{C}_{+},
\end{array}\right.
$$

where $a=\frac{A}{2} \geqq 2$. The differential of $T_{A},\left[\begin{array}{cc}0 & 1 \\ -1 & 2 \pm A\end{array}\right]=\mathscr{M}_{ \pm}$, is a hyperbolic matrix (i.e. with eigenvalues outside the unit circle) in $\mathscr{B}_{+}$for all $A>0$ and in $\mathscr{B}_{-}$for $A>4$. The boundary directions of the sectors above are defined by the expanding eigenvectors of the matrices $\mathscr{M}_{ \pm}$. Such a choice of sectors is optimal for further considerations. Moreover this remark enables us to prove the following lemma without computations.

Lemma 1. $D T_{A}(U(p)) \subset U\left(T_{A} p\right)$.

Lemma 2. Let $v=\left(\xi_{1}, \xi_{2}\right) \in U(p)$ then

$$
\begin{array}{lll}
\left\|D T_{A} v\right\| \geqq(a-1+\sqrt{a(a-2)})\|v\| & \text { if } & p \in \mathscr{B}_{-} \cap \mathscr{C}_{-} \\
\left\|D T_{A} v\right\| \geqq 2(a-1)\|v\| & \text { if } & p \in \mathscr{B}_{-} \cap \mathscr{C}_{+} \\
\left\|D T_{A} v\right\| \geqq 2(a+1)\|v\| & \text { if } & p \in \mathscr{B}_{+} \cap \mathscr{C}_{-} \\
\left\|D T_{A} v\right\| \geqq(a+1+\sqrt{a(a+2)})\|v\| & \text { if } & p \in \mathscr{B}_{+} \cap \mathscr{C}_{+} .
\end{array}
$$

The proof of the last lemma is obtained by straightforward calculations. Lemmas 1 and 2 show that when $A>4$ the matrices $\mathscr{M}_{ \pm}$are compatibly hyperbolic, i.e. a product of any number of these matrices in any order is also hyperbolic.

Theorem 3. If $A \geqq 4$ then $T_{A}$ is almost hyperbolic in the whole torus.

Proof. For $A>4$ this follows directly from Lemmas 1, 2, and Theorem 2.

In the case $A=4$ we do not have sufficient expansion of vectors on $\mathscr{B}_{-} \cap \mathscr{C}_{-}$. So we take $\mathscr{B}_{+}$for $\mathscr{K}$ in Theorem 2 and we have only to show that $\bigcup_{i=-\infty}^{+\infty} T_{4}^{i} \mathscr{B}_{+}$has full measure, or equivalently that almost all points from $\mathscr{B}_{-}$leave $\mathscr{B}_{\text {_. This follows }}$ from the structure of $T_{4}^{2}$ on $\mathscr{B}_{-} \cap T_{4}^{-1}\left(\mathscr{B}_{-}\right): T_{4}^{2}$ preserves the family of lines $x_{1}+x_{2}=$ const and on each line $T_{4}^{2}$ acts as a nontrivial translation, except for the line $x_{1}+x_{2}=-\frac{1}{2}$ which passes through the fixed point $\left(-\frac{1}{4},-\frac{1}{4}\right)$.

Theorem 4. If $A>A_{0}$ where $t_{0}=\frac{A_{0}}{2}$ is the positive solution of the equation $t-\sqrt{t(t-2)}+\frac{1}{2(t+1)}=2$, then $T_{A}$ and all its powers are ergodic and hence $T_{A}$ is Bernoulli $\left(A_{0} \approx 4.0329\right)$. 
In the proof we shall use the following lemmas.

Lemma 3. Let $r, s \geqq 0$ then

$$
\inf _{0 \leqq \mu \leqq 1}(\max (r \mu, s(1-\mu)))=\frac{1}{r^{-1}+s^{-1}}=\frac{r s}{r+s} .
$$

By $\ell(\cdot)$ we will denote the length of an arc defined by the norm $\|\cdot\|$.

Lemma 4. Let $A>A_{0}$ where $A_{0}$ is as in Theorem 4. Then there is $h>0$ such that if $\gamma^{u}(p)$ crosses the singularity lines $\partial \mathscr{B}_{ \pm}$at most once then $T_{A} \gamma^{u}(p)$ contains a segment $\gamma^{u}(q), q \in T_{A} \gamma^{u}(p)$ such that

$$
\ell\left(\gamma^{u}(q)\right) \geqq(1+h) \ell\left(\gamma^{u}(p)\right) .
$$

Proof. By the construction of local expanding fibres it follows that $\gamma^{u}(p)$ is contained either in $\mathscr{C}_{-}$or in $\mathscr{C}_{+}$. Suppose $\gamma^{u}(p)$ intersects the singularity lines $\partial \mathscr{B}_{ \pm}$ at most once. Then we can write $\ell\left(\gamma^{u}(p) \cap \mathscr{B}_{-}\right)=\mu \ell\left(\gamma^{u}(p)\right)$ and $\ell\left(\gamma^{u}(p) \cap \mathscr{B}_{+}\right)$ $=(1-\mu) \ell\left(\gamma^{u}(p)\right)$ where $0 \leqq \mu \leqq 1$. So by Lemma 2

$$
\begin{aligned}
& \ell\left(T_{A}\left(\gamma^{u}(p) \cap \mathscr{B}_{-}\right)\right) \geqq \begin{cases}(a-1+\sqrt{a(a-2)}) \mu \ell\left(\gamma^{u}(p)\right) & \text { if } \gamma^{u}(p) \subset \mathscr{C}_{-} \\
2(a-1) \mu \ell\left(\gamma^{u}(p)\right) & \text { if } \gamma^{u}(p) \subset \mathscr{C}_{+}\end{cases} \\
& \ell\left(T_{A}\left(\gamma^{u}(p) \cap \mathscr{B}_{+}\right)\right) \geqq \begin{cases}2(a+1)(1-\mu) \ell\left(\gamma^{u}(p)\right) & \text { if } \gamma^{u}(p) \subset \mathscr{C}_{-} \\
(a+1+\sqrt{a(a+2)})(1-\mu) \ell\left(\gamma^{u}(p)\right) & \text { if } \gamma^{u}(p) \subset \mathscr{C}_{+}\end{cases}
\end{aligned}
$$

Hence the lemma will be proved whenever

$$
\inf _{0 \leqq \mu \leqq 1}(\max ((a-1+\sqrt{a(a-2)}) \mu, 2(a+1)(1-\mu)))>1
$$

and

$$
\inf _{0 \leqq \mu \leqq 1}(\max (2(a-1) \mu,(a+1+\sqrt{a(a+2)})(1-\mu)))>1 .
$$

According to Lemma 3 this is equivalent to

$$
a-1-\sqrt{a(a-2)}+\frac{1}{2(a+1)}<1
$$

and

$$
\frac{1}{2(a-1)}+\frac{1}{a+1+\sqrt{a(a+2)}}<1 .
$$

The second inequality certainly holds for all $A \geqq 4$ and the first for $A>A_{0}$ where $A_{0}$ is described in Theorem 4.

Proof of Theorem 4. We will prove that sufficiently distant images of an arbitrary local expanding fiber $\gamma^{u}(p)$ contain segments crossing the square $\mathscr{B}_{+} \cap \mathscr{C}_{+}$from the lower to the upper side. $S$-reversibility implies that sufficiently distant preimages of a local contracting fiber contain segments crossing the square $\mathscr{B}_{+} \cap \mathscr{C}_{+}$from the left to the right side. So applying Theorem 1 we obtain our theorem. 
The heart of the matter lies in the fact that $T_{A}^{k} \gamma^{u}(p)$ is a broken line with sharp angles between consecutive segments (because $D T_{A}$ in $\mathscr{B}_{-}$changes the orientation of expanding sectors) so that although its length tends to infinity with $k$ it could in principle be confined to a small region.

From Lemma 4 it follows that for sufficiently large $k \geqq 0 T_{A}^{k} \gamma^{u}(p)$ contains a segment $\gamma^{u}\left(p_{1}\right), p_{1} \in T_{A}^{k} \gamma^{u}(p)$ which crosses the singularity lines $\partial \mathscr{B}_{ \pm}$at least twice. Hence $\gamma^{u}\left(p_{1}\right)$ crosses one of the four squares $\mathscr{B}_{+} \cap \mathscr{C}_{+}, \mathscr{B}_{+} \cap \mathscr{C}_{-}, \mathscr{B}_{-} \cap \mathscr{C}_{+}, \mathscr{B}_{-} \cap \mathscr{C}_{-}$ from the lower to the upper side.

In the first case we are done, because by Lemma 2 the length of $\gamma^{u}\left(p_{1}\right)$ is expanded at least $3+2 \sqrt{2}$ times so the image of $\gamma^{u}\left(p_{1}\right) \cap \mathscr{B}_{+} \cap \mathscr{C}_{+}$also crosses $\mathscr{B}_{+} \cap \mathscr{C}_{+}$.

In the second case [i.e. $\gamma^{u}\left(p_{1}\right)$ crosses $\mathscr{B}_{+} \cap \mathscr{C}_{-}$], we find that $T_{A} \gamma^{u}\left(p_{1}\right)$ contains a segment in $\mathscr{C}_{+}$whose length is at least 3 because in $\mathscr{B}_{+} \cap \mathscr{C}_{-}$the rate of expansion is at least 6. So it must cross the square $\mathscr{B}_{+} \cap \mathscr{C}_{+}$.

In the third case [i.e. $\gamma^{u}\left(p_{1}\right)$ crosses $\mathscr{B}_{-} \cap \mathscr{C}_{+}$] by the same argument $T_{A} \gamma^{u}\left(p_{1}\right)$ must contain a segment in $\mathscr{B}_{+} \cap \mathscr{C}_{-}$with the length greater than $\frac{1}{4}$. Hence $T_{A}^{2} \gamma^{u}\left(p_{1}\right)$ contains a segment in $\mathscr{C}_{+}$whose length is greater than $\frac{3}{2}-$ so it crosses $\mathscr{B}_{+} \cap \mathscr{C}_{+}$.

The last case, when $\gamma^{u}\left(p_{1}\right)$ crosses $\mathscr{B}_{-} \cap \mathscr{C}_{-}$, needs more careful consideration because in $\mathscr{B}_{-} \cap \mathscr{C}_{-}$the rate of expansion is very close to one. The center of $\mathscr{B}_{-} \cap \mathscr{C}_{-}$is a fixed point $c_{0}=\left(-\frac{1}{4},-\frac{1}{4}\right)$ which is hyperbolic for $A>4$. Its local contracting fiber $\gamma^{s}\left(c_{0}\right)$ crosses $\mathscr{B}_{-} \cap \mathscr{C}_{-}$from the left side to the right side. Hence $\gamma^{u}\left(p_{1}\right)$ intersects $\gamma^{s}\left(c_{0}\right)$. It follows that images of $\gamma^{u}\left(p_{1}\right)$ approach $\gamma^{u}\left(c_{0}\right)$ (more exactly contain segments arbitrarily close to $\left.\gamma^{u}\left(c_{0}\right)\right)$. So it suffices to prove that images of $\gamma^{u}\left(c_{0}\right)$ cross $\mathscr{B}_{+} \cap \mathscr{C}_{+}$from the lower to the upper side.

Denote by $c_{\ell}$ and $c_{r}$ respectively the left and the right ends of $\gamma^{u}\left(c_{0}\right)$. The segment $\overline{c_{0} c_{\ell}}$ crosses the singularity lines only once and hence by Lemma 4 its image under $T_{A}$ must contain a segment with greater length. But $T_{A}\left(\overline{c_{0} c_{\ell}}\right)$ $=\overline{c_{0} c_{r}} \cup \overline{c_{r} T_{A}\left(c_{\ell}\right)}$ and hence $\left.\ell\left(\overline{c_{r} T_{A}\left(c_{\ell}\right.}\right)\right)>\ell\left({\overline{c_{0} c_{r}}}_{r}\right)=\ell\left(\overline{c_{0} c_{\ell}}\right)$.

This fact together with some calculations shows that the segment $\overline{c_{r} T_{A}\left(c_{\ell}\right)}$ crosses the opposite sides of the parallelogram $T_{A}^{-1}\left(\mathscr{B}_{-} \cap \mathscr{C}_{-}\right)$(see Fig. 1). So $\left.T_{A}\left(\overline{c_{r} T_{A}\left(c_{\ell}\right.}\right)\right)$ contains a segment which crosses $\mathscr{B}_{-} \cap \mathscr{C}_{-}$from the lower to the upper side. Denote by $\overline{c_{1} c_{2}}$ the segment in the intersection of the latter segment and $T_{A}^{-1}\left(\mathscr{B}_{+} \cap \mathscr{C}_{-}\right)$as shown in Fig. 1 . It is easy to see that $\ell\left(T_{A}\left(\overline{c_{1} c_{2}}\right)\right)>\frac{1}{4}$. Further by Lemma $2 \ell\left(T_{A}^{2}\left(\overline{c_{1} c_{2}}\right)\right)>\frac{3}{2}$ and so $T_{A}^{2}\left(\overline{c_{1} c_{2}}\right)$ must cross $\mathscr{B}_{+} \cap \mathscr{C}_{+}$.

I do not doubt that $T_{A}$ is ergodic for all $A \geqq 4$, but apparently a different approach is needed to prove this.

\section{Ergodic Properties of $T_{A}$ for $A<4$}

For $A<4$ the matrix $\mathscr{M}_{-}$which represents $D T_{A}$ in $\mathscr{B}_{-}$becomes elliptic - i.e. has eigenvalues on the unit circle different from 1 and -1 . It is convenient to introduce coordinates $\left(y_{1}, y_{2}\right)$ in which it will be represented by the rotation matrix

$$
y_{1}=x_{1}-\cos \beta x_{2}, y_{2}=\sin \beta x_{2} \text { where } \cos \beta=1-a \text {. }
$$

In these coordinates $T_{A}$ considered on $\mathbb{R}^{2}$ rotates the strip $\mathscr{B}_{-}$by the angle $-\beta$ around the point $\left(\frac{\cos \beta-1}{4},-\frac{\sin \beta}{4}\right)$. 


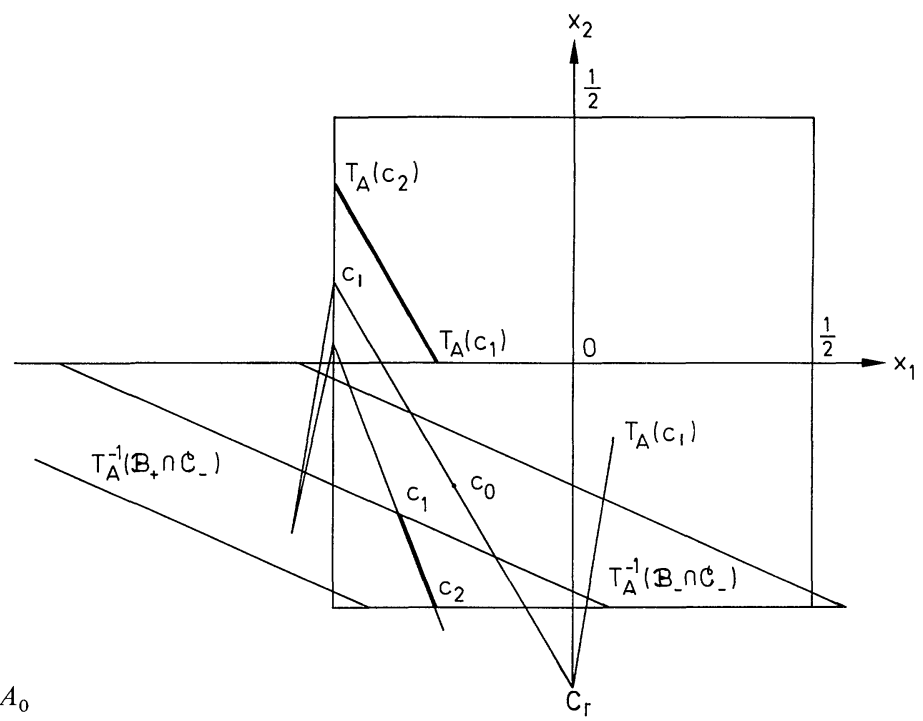

Fig. 1. $A>A_{0}$

Let $\mathscr{D}=\left\{p \mid T_{A}^{k} p \in \mathscr{B}_{-}\right.$for all $\left.k\right\}$. By $S$-reversibility we have $S \mathscr{D}=S \bigcap_{k=-\infty}^{+\infty} T_{A}^{k} \mathscr{B}$ $=\bigcap_{k=-\infty}^{+\infty} T_{A}^{-k} S \mathscr{B}_{-}=\bigcap_{k=-\infty}^{+\infty} T_{A}^{-k} T_{A} \mathscr{B}_{-}=\mathscr{D}$. It is easy to see that in $y$-coordinates $\mathscr{D}$ is a disk inscribed in the rhombus $\mathscr{B}_{-} \cap \mathscr{C}_{-}$whenever the angle of rotation $\beta$ is irrational. If $\beta=\frac{p}{q} \pi$ then $\mathscr{D}$ is a $2 q$-sided regular polygon with four sides on the sides of $\mathscr{B}_{-} \cap \mathscr{C}_{-} . T_{A}$ in $\mathscr{D}$ has period $q$ for $p$ even and $2 q$ for $p$ odd.

Moreover $\mathscr{D}=\left\{p \mid T_{A}^{k} p \in \mathscr{B}_{\text {_ }}\right.$ for $\left.k \geqq 0\right\}$.

In $x$-coordinates the disk becomes an ellipse with axes on the diagonals of $\mathscr{B}_{-} \cap \mathscr{C}_{-}$. The polygon in $x$-coordinates is no longer regular but it is symmetric about its center and has two axes of symmetry - the diagonals of $\mathscr{B}_{-} \cap \mathscr{C}_{-}$.

Theorem 5. For $A=2\left(\cos \frac{\pi}{n}+1\right), n=2,3, \ldots$, there is a $2 n$-sided convex polygon $\mathscr{D} \subset \mathscr{B}_{-} \cap \mathscr{C}_{-}$such that $T_{A}$ is almost hyperbolic in $\mathbb{T}^{2} \backslash \mathscr{D}$. Moreover $\mathscr{D}$ is symmetric about its center $\left(-\frac{1}{4},-\frac{1}{4}\right)$ and with respect to the diagonals of $\mathscr{B}_{-} \cap \mathscr{C}_{-}$. The points $\left(-\frac{1}{2}, 0\right)$ and $\left(0,-\frac{1}{2}\right)$ are common vertices of $\mathscr{D}$ and $\mathscr{B}_{-} \cap \mathscr{C}_{-} . T_{A}$ in $\mathscr{D}$ has period $n$ for $n$ odd and period $2 n$ for $n$ even.

In the tangent space we consider coordinates $\eta_{i}=d y_{i}, i=1,2$, and the norm $\left\|\left(\eta_{1}, \eta_{2}\right)\right\|=\sqrt{\eta_{1}^{2}+\eta_{2}^{2}}$.

Now $D T_{A}$ in $\mathscr{B}_{-}$is given by the matrix of rotation by the angle $-\beta$ $\mathscr{M}_{1}=\left[\begin{array}{cc}\cos \beta & \sin \beta \\ -\sin \beta & \cos \beta\end{array}\right]$ and in $\mathscr{B}_{+}$by the matrix $\mathscr{M}_{3}, \mathscr{M}_{3}=\mathscr{M}_{1} \mathscr{M}_{2}$ where $\mathscr{M}_{2}=\left[\begin{array}{cc}1 & -4 \tan \frac{\beta}{2} \\ 0 & 1\end{array}\right]$. 
So $D\left(T_{A}^{k}\right)$ on $\mathscr{B}_{+}$has the form $\mathscr{M}_{1}^{n_{\ell}} \mathscr{M}_{2} \ldots \mathscr{M}_{2} \mathscr{M}_{1}^{n_{1}} \mathscr{M}_{2}, \sum_{i=1}^{\ell} n_{i}=k$.

In the tangent space consider the sectors

$$
\begin{aligned}
& U=\left\{\left(\eta_{1}, \eta_{2}\right) \mid \frac{\eta_{1}}{\eta_{2}} \leqq 2 \tan \frac{\beta}{2}\right\} \\
& U_{1}=\mathscr{M}_{2} U=\left\{\left(\eta_{1}, \eta_{2}\right) \mid \frac{\eta_{1}}{\eta_{2}} \leqq-2 \tan \frac{\beta}{2}\right\} .
\end{aligned}
$$

Lemma 5. The only $A$ for which $\mathscr{M}_{1}^{k} U_{1} \subset U$ for all $k \geqq 0$ are $A=2\left(\cos \frac{\pi}{n}+1\right)$.

Proof. Obviously the angle $\beta$ must be rational, i.e. $\beta=\frac{p}{q} \pi$. Let $\mathscr{R}$ be the matrix of rotation by the angle $-\frac{q-1}{q} \pi$. The required property is now equivalent to $\mathscr{R} U_{1} \subset U$. It is easy to see that the latter is equivalent to $2 \gamma<\frac{1}{q} \pi$ where $\gamma$ is the angle of the sector $U_{1}$.

But $\tan \gamma=\left(2 \tan \frac{\beta}{2}\right)^{-1}=\left(2 \tan \frac{p}{2 q} \pi\right)^{-1}$ so we must have $\tan \frac{\pi}{2 q} \cdot \tan \frac{p \pi}{2 q} \geqq \frac{1}{2}$. For $p=q-1$ the lefthand side is equal to 1 , so the inequality holds. For $p \leqq q-2 \tan \frac{\pi}{2 q} \cdot \tan \frac{p \pi}{2 q} \leqq \tan \frac{\pi}{2 q} \cdot \tan \frac{(q-2) \pi}{2 q}=\frac{\tan \frac{\pi}{2 q}}{\tan \frac{\pi}{q}}<\frac{1}{2}$.

Proof of Theorem 5. We apply Theorem 2 . We consider $\mathscr{B}_{+}$with constant sector bundle equal to $U$. By what was said in the beginning of this section, $\bigcup_{i=-\infty}^{+\infty} T_{A}^{i} \mathscr{B}_{+}$ $=\mathbb{T}^{2} \backslash$ int $\mathscr{D}$. Property (i) follows from Lemma 5 .

$U$ consists of vectors which are not decreased in norm by $\mathscr{M}_{2} . \mathscr{M}_{1}$ preserves the norm of vectors. So the first part of property (ii) holds.

$\mathscr{M}_{2} \mathscr{M}_{1}^{k} \mathscr{M}_{2}$ increases the norm of vectors from $U$ for all $k$ except for multiples of $n$.

For $p \in \mathscr{B}_{-} \backslash \mathscr{D}$ by $k(p)$ denote the time which $p$ stays in $\mathscr{B}_{-}$i.e. $T_{A}^{i} p \in \mathscr{B}_{-}$for $0 \leqq i<k(p)$ and $T_{A}^{k(p)} p \notin \mathscr{B}$. . It is easy to see that $k(p)$ can be equal to $1,2, \ldots, n-1$ (see Fig. 2). The set $Q=\left\{p \in \mathscr{B}_{-}|\mathscr{D}| k(p)=n-1\right\}$ consists of two triangles as depicted in Fig. 2. $T_{A}^{n}$ for $n$ odd and $T_{A}^{2 n}$ for $n$ even act on $Q$ in the $x$-coordinates as a rotation of the circles $x_{1}=$ const by a changing angle. It follows that the measure of points which always stay in $\mathscr{B}_{+}$once and enter $\mathscr{B}_{-}$via the set $Q$ is equal to zero. So for almost all points from $\mathscr{B}_{+}$the vectors from $U$ are eventually increased. Hence our theorem follows from Theorem 2.

We conjecture that for $2 \leqq A<4$ D is the only elliptic island. But it seems possible that for some $A$ the metric entropy of $T_{A}$ is zero.

In the end we will consider the case $A=1$. We have two closed invariant curves which are broken lines with three segments. One of them has vertices in $\left(0,-\frac{1}{2}\right)$, $\left(\frac{1}{4}, 0\right),\left(\frac{1}{2}, \frac{1}{4}\right)=\left(-\frac{1}{2}, \frac{1}{4}\right),\left(0, \frac{1}{2}\right)$ (in $x$-coordinates). The other is symmetric under $S$. These curves divide the torus into two invariant parts $M_{1}$ and $M_{2}$ (Fig. 3). In $M_{1}$ we have the elliptic island $\mathscr{D}$ which is a hexagon. 


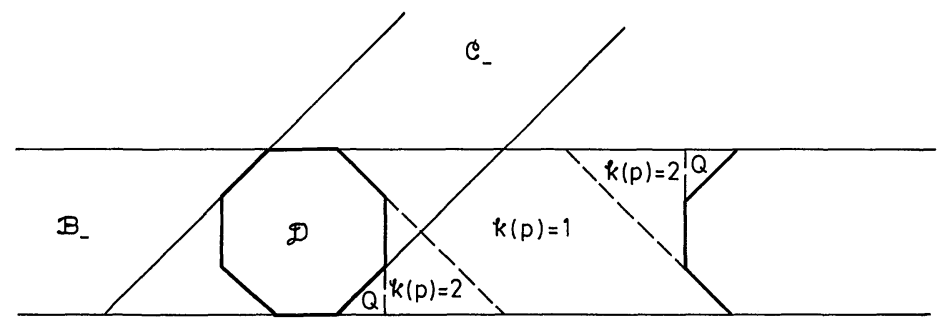

Fig. 2. $A=2+\sqrt{2}$, in $y$-coordinates

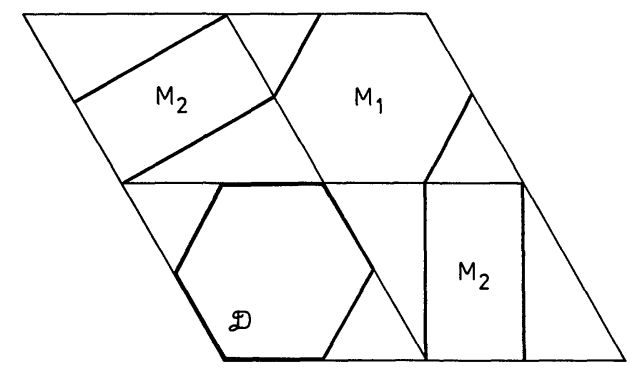

Fig. 3. $A=1$, in $y$-coordinates

Theorem 6. $T_{1}$ is almost hyperbolic in $M_{1} \backslash \mathscr{D}$.

Proof. Consider the sectors

$$
U=\left\{\left(\eta_{1}, \eta_{2}\right) \mid \frac{\eta_{1}}{\eta_{2}} \leqq \sqrt{3}\right\}
$$

and

$$
U_{1}=\left\{\left(\eta_{1}, \eta_{2}\right) \mid-\frac{1}{\sqrt{3}} \leqq \frac{\eta_{1}}{\eta_{2}} \leqq 0\right\} \supset \mathscr{M}_{3}^{2} U .
$$

One checks easily that $\mathscr{M}_{1} U_{1} \subset U$ and $\mathscr{M}_{3} \mathscr{M}_{1}^{2} U_{1} \subset U_{1}$.

We apply Theorem 2 taking $\mathscr{K}=M_{1} \cap \mathscr{B}_{+} \cap T_{1}^{-1} \mathscr{B}_{+}$and the constant sector bundle equal to $U$.

The crucial remark is the following: if a point enters $M_{1} \cap \mathscr{B}_{-}$and then immediately leaves it then it must stay in $M_{1} \cap \mathscr{B}+$ at least for time 2 , i.e. enter $\mathscr{K}$. Since a point can stay in $M_{1} \cap \mathscr{B}$ - only for time 1 or 2 , it follows that the property (i) holds.

Vectors from $U$ are not decreased by $\mathscr{M}_{3}^{2}$ [so formally should we rather consider $T_{1}^{2}$ to obtain the first part of the property (ii)]. $\mathscr{M}_{3} \mathscr{M}_{1}^{2} \mathscr{M}_{3}^{2}$ increases the norm of all vectors from $U$. So the situation that the boundary vector of $U$ is never increased, can only take place if $D\left(T_{1}^{3 k}\right)$ is equal to $\left(\mathscr{M}_{1} \mathscr{M}_{3}^{2}\right)^{k}$ for all $k \geqq 0$. Simple geometric considerations show that the set of points with the above property has measure zero. Also $\bigcup_{i=-\infty}^{+\infty} T_{1}^{i} \mathscr{K}$ is almost equal to $M_{1} \backslash \mathscr{D}$.

In $M_{2}$ the picture is more complicated. The periodic orbit of period $2\left\{\left(-\frac{1}{4}, \frac{1}{4}\right)\right.$, $\left.\left(\frac{1}{4},-\frac{1}{4}\right)\right\}$ is elliptic. (It is elliptic for $0<A<2$.) The corresponding elliptic islands are 


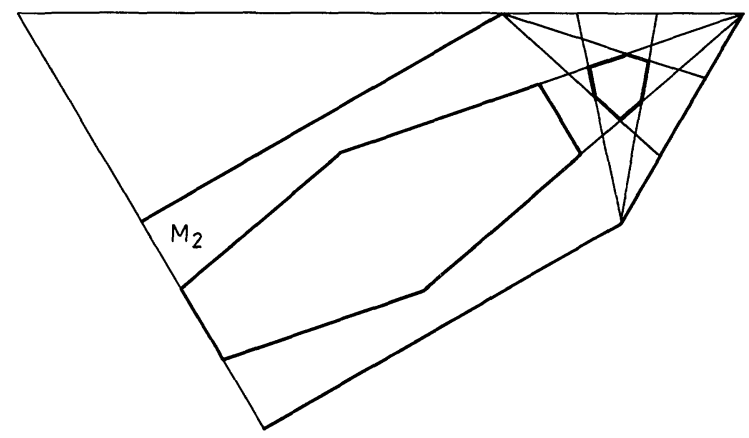

Fig. 4. Elliptic islands in $M_{2}$, in $y$-coordinates

hexagons symmetric under $S$. [One of them has vertices in $\left(-\frac{1}{4}, \frac{1}{6}\right),\left(0, \frac{1}{3}\right),\left(0, \frac{5}{12}\right)$, $\left(-\frac{1}{4}, \frac{1}{3}\right),\left(-\frac{1}{2}, \frac{1}{6}\right),\left(-\frac{1}{2}, \frac{1}{12}\right)$ (Fig. 4).]

Furthermore there are two elliptic periodic orbits of period 5 . One of them is the orbit of the point $\left(\frac{1}{12}, \frac{5}{12}\right)$, the other is symmetric under $S$. The island around $\left(\frac{1}{12}, \frac{5}{12}\right)$ has vertices in $\left(\frac{1}{16}, \frac{3}{8}\right),\left(\frac{1}{10}, \frac{2}{5}\right),\left(\frac{1}{8}, \frac{7}{16}\right),\left(\frac{1}{10}, \frac{9}{20}\right),\left(\frac{1}{16}, \frac{7}{16}\right),\left(\frac{1}{20}, \frac{2}{5}\right)$ (Fig. 4).

There is also a rather degenerate phenomenon: a quadrilateral of periodic points with period 33 which is not an elliptic island. (There is no point in it with lower period.)

The question of mixing properties of $T_{1}$ in $M_{2}$ is open. Also the behaviour of the invariant curves with the change of $A$ is unclear; computer experiments indicate that they disappear immediately for $A<1$ and persist for $A>1$.

Thus Theorems 5 and 6 provide examples with coexistence of stochastic and integrable behaviour. Perhaps these results have some significance for the case of smooth perturbations of the twist mapping.

Acknowledgement. The author thanks Prof. Z. Nitecki who read the manuscript and contributed to its improvement.

\section{References}

1. Katok, A.: Bernoulli diffeomorphisms on surfaces. Preprint, IHES/M/78/232, France (1978)

2. Wojtkowski, M.: Linked twist mappings have the $K$-property. Annals of the New York Academy of Sciences, Vol. 357. Nonlinear Dynamics 65-76 (1980)

3. Grzegorczyk, P.: Master's thesis. Warsaw University (1980)

4. Zaslavski, G.M.: Statistical irreversibility in nonlinear systems, Chap. 4. Moscow: Nauka 1970

5. Katok, A., Sinai, Ya.G., Stepin, A.M.: Math. Anal. 13, 129-262 (1975)

6. Devaney, R.L.: Trans. Am. Math. Soc. 218, 89-113 (1976)

7. Sinai, Ya.G.: Izv. Akad. Nauk SSSR 30, No. 1, 15-68 (1966); Math. USSR Izv. 30, 15-68 (1966)

8. Weiss, B.: The geodesic flow on surfaces of negative curvature. In : Lecture notes in physics, Vol. 38. Berlin, Heidelberg, New York: Springer 1975

9. Ornstein, D.S. : Ergodic theory, randomness and dynamical systems. Yale: Yale University Press 1974

Communicated by D. Ruelle

Received August 29, 1980; in revised form December 29, 1980 